\title{
Rejets diphasiques accidentels et calcul d'évents pour la protection des installations dans l'industrie chimique
}

\author{
Accidental two-phase releases and vent-sizing for vessel protection \\ in the chemical industry
}

par Jean-Louis Gustin

Rhône-Poulenc Industrialisation

The most severe accidents occurred in the chemical industry (Bhopal, Seveso, Griesheim) due to runaway reactions were not caused by the reactor explosion. A two-phase release of toxic material was sprayed over a densely populated area, through a vent system.

In the above three examples, the vents were not sized for the accident scenario.

The presence of large vents (pressure safety valves, rupture disks) to protect industrial equipments, is a source of potential two-phase release, should an accidental blow-down occur, if there is no catchment system. The occurrence of a two-phase release may be predicted using vapor/liquid disengagement correlations. When the DIERS methodology is applied for vent-sizing to protect vessels for the runaway reaction case, the occurrence of a two-phase release is considered. The two-phase release must be directed to a catchment system to avoid a pollution of the environment.

This paper gives an overview of the DIERS methodology applied to vent sizing for runaway reactions, with special attention to the areas/topics still discussed.

The influence of the cost and reliability of catchment/release treatment/disposal systems on process design and on protection using safety interlocks is discussed.

\section{INTRODUCTION}

Les accidents les plus sévères survenus dans l'industrie chimique, liés à des emballements de réactions, ne sont pas le résultat de l'explosion d'un réacteur ou d'une capacité. La pressurisation engendrée par l'emballement de réaction a été limitée par l'ouverture d'un évent de secours. La dépressurisation rapide de l'enceinte s'est accompagnée d'un entraînement notable du liquide qu'elle contenait.

La taille de l'évent était suffisante pour éviter l'éclatement de l'enceinte, bien que celui-ci n'ait pas été dimensionné spécifiquement pour ce scénario. La sévérité de ces accidents est liée aux propriétés toxiques des substances ainsi rejetées dans l'environnement et aux zones concernées par les retombées solides ou liquides. Les accidents de Bhopal (1984), Seveso (1976) et Griesheim (1993) mentionnés seulement parce qu'ils sont bien documentés dans la littérature, prouvent que les systèmes d'évents de secours ne doivent pas seulement prévenir l'éclatement des enceintes qui sont le siège d'un emballement thermique, mais aussi assurer un traitement fiable du rejet.
Le but de la sécurité des procédés dans le domaine des emballements de réactions n'est pas seulement d'empêcher l'explosion des appareils, il est aussi de concevoir des installations qui ne mettent pas en danger les populations avoisinantes et ne causent pas de dommages graves à l'environnement.

Dans les trois accidents mentionnés, le système d'évent n'était pas dimensionné pour le scénario de l'emballement thermique à l'origine de l'accident et les hypothèses de dimensionnement n'étaient pas spécifiées. Cependant, on doit se rendre à l'évidence que des évents de grande taille, disques de rupture ou soupapes, disposés sur des réacteurs ou des stockeurs, dimensionnés pour le cas incendie ou tout autre scénario, sans un système de captage/traitement du rejet, sont des sources potentielles de rejets diphasiques gaz/liquide si un emballement de réaction survient dans l'enceinte qu'ils protègent.

Un rejet diphasique peut également résulter de la dépressurisation accidentelle d'une enceinte contenant un liquide pressurisé par un gaz soluble comme $\mathrm{CO}_{2}, \mathrm{H}_{2}$, etc...

Les accidents ont montré qu'un rejet diphasique gaz/liquide de l'encours d'une enceinte, pulvérisé sur le voisinage n'est pas acceptable dans des zones densément peu- 
plées. Un système de captage du rejet est nécessaire lorsque les corrélations de désengagement gaz/liquide montrent que l'entraînement du liquide est probable.

La toxicité du rejet peut être prise en compte pour évaluer la nécessité d'un système de captage. Cependant, il ne suffit pas de tenir compte de la toxicité des réactifs et des produits normaux du procédé considéré. Les produits de réactions indésirables, inattendues ou inconnues qui se trouvent initiées lors des emballements de réactions doivent être pris en compte.

L'analyse des produit finals recueillis lors d'accidents liés à des emballements de réactions comme ceux qui sont mentionnés, a montré que des produits différents des produits habituels du procédé sont obtenus. Ce type d'analyse très coûteuse est seulement réalisé lors des enquêtes d'accidents. Les produits possibles des emballements de réactions restent pour l'essentiel inconnus et dépendent beaucoup du scénario de l'emballement et des conditions du rejet.

Il est raisonnable de penser que le rejet diphasique est potentiellement toxique et que, de ce fait, un système de captage du rejet serait nécessaire.

L'installation de systèmes de captage/traitement du rejet est envisagée sur des évents disposés sur des réacteurs. Cependant, des emballements de réactions sont également possibles dans des stockeurs, les bouilleurs de colonnes à distiller, des conduites, des conteneurs utilisés pour le transport de produits chimiques. Si l'on voulait adopter une protection par évents de tous ces équipements, il faudrait créer des réseaux complexes d'évents de secours qui devraient être reliés à un grand nombre de systèmes de captage/traitement de rejets dans les usines chimiques.

Si l'on considère encore les accidents mentionnés plus haut, il apparaît aussi que les évents existants n'étaient pas dimensionnés pour le scénario de l'accident et se sont ouverts occasionnellement pendant l'emballement thermique qu'ils n'ont pas forcément bien contrôlé ou dont ils ont aggravé les conséquences.

Des systèmes d'évents peuvent être adaptés pour un scénario donné et avoir un effet défavorable dans des circonstances différentes.

Cette situation peut en effet se présenter dans les ateliers polyvalents étudiés et construits sur une base standardisée sans connaître les produits qui vont être fabriqués dans l'installation pendant sa durée de vie.

Cette situation est fréquente dans la chimie de spécialités, la chimie fine et la pharmacie et cela est plus rare dans la pétrochimie, la fabrication de plastiques et de polymères où le même produit va être fabriqué dans l'installation indéfiniment.

Depuis de nombreuses années l'industrie chimique a évolué de la fabrication de "commodités", produits de gros tonnage à bas prix vers les fabrications de chimie fine à forte valeur ajoutée. Cela induit une demande accrue pour des études de sécurité des procédés concernant des fabrications de chimie fine, dans des procédés présentant des étapes nombreuses et complexes mais avec des encours de matières faibles.

Dans ce type de chimie, la sévérité des accidents liés à des emballements de réactions est moindre que dans la chimie lourde, cependant la probabilité d'avoir des incidents est plus grande du fait des réactions chimiques et des molécules plus complexes impliquées. Dans ce cas, le but de la sécurité des procédés est davantage de prévenir les accidents liés à des emballements de réactions que d'en limiter les conséquences.

Si l'on considère les procédés de chimie lourde les plus répandus, il n'y a pas de raison que les mêmes précautions ne soient pas prises pour la prévention des accidents, en éliminant les causes connues des emballements de réactions.

La mise au point de procédés fiables dans lesquels les incidents liés à des emballements de réactions ont été réduits ou éliminés, constitue une solution économiquement viable comparée au contrôle et à la limitation des conséquences d'emballements thermiques sévères au moyen de systèmes d'évent munis de dispositifs de captage des rejets.

L'étude des scénarios d'emballement thermique en vue d'obtenir les données nécessaires au calcul d'évents permet l'identification des dérives dangereuses des procédés. Cela permet le choix de la version la plus fiable du procédé et la prévention des dérives dangereuses par des dispositifs de sécurité, limitant de ce fait le recours à des systèmes d'évent de grande taille.

\section{II — PROTECTION DES INSTALLATIONS CHIMIQUES PAR ÉVENT POUR LE CONTRÔLE DES EMBALLEMENTS THERMIQUES}

Les évents de secours sont disposés sur des appareils ou capacités (réacteurs, colonnes à distiller, bouilleurs, stockeurs...) contenant des substances ou mélanges susceptibles de subir une décomposition thermique ou un emballement de réaction, afin d'empêcher l'éclatement ou l'explosion de ces équipements.

En principe, ces appareils ou stockeurs ne peuvent pas résister à la pression générée par l'emballement de réaction.

Le Design Institute for Emergency Relief Systems (DIERS), un groupe de travail de l'AIChE a publié des recommandations pour le calcul d'évents qui prennent en compte l'émission d'un écoulement diphasique gaz/liquide lorsque l'évent s'ouvre.

Les méthodes connues jusque là, établies par l'API, prenant en compte seulement l'émission d'un rejet gazeux, pouvaient conduire à un sous-dimensionnement des évents d'un facteur 6 à 10 si un emballement de réaction était initié dans l'appareil protégé [1]. La méthodologie du DIERS pour le calcul d'évents comprend les étapes suivantes :

$1^{\text {re }}$ étape : Définition du scénario majorant d'emballement de réaction servant de base au dimensionnement d'évent.

$2^{\mathrm{e}}$ étape : Caractérisation du comportement du système réactionnel au moyen de techniques calorimétriques pseudo-adiabatiques. Les systèmes réactionnels sont divisés en 3 catégories :

- les systèmes à tension de vapeur,

- les réactions produisant des gaz incondensables,

- les systèmes hybrides.

$3^{\mathrm{c}}$ étape : Acquisition des données expérimentales nécessaires au calcul d'évent. La nature des données nécessaires dépend du type de système réactionnel. Les données expérimentales doivent être obtenues dans des conditions voisines de l'adiabatique pour obtenir une simulation expérimentale correcte de l'emballement thermique.

$4^{\mathrm{e}}$ étape : Choix des méthodes de calcul d'évent et de calcul des écoulements diphasiques en fonction du type de système réactionnel.

\subsection{Caractérisation des systèmes réactionnels}

Dans la classification du DIERS, il existe 3 types de systèmes réactionnels.

\subsubsection{Systèmes à forte tension de vapeur}

Les systèmes à forte tension de vapeur sont des systèmes réactionnels dans lesquels la pressurisation des enceintes est due à un équilibre liquide/vapeur. La tension de vapeur peut 
être celle de solvants, de réactifs, de produits ou du mélange réactionnel dans son ensemble.

Les systèmes à forte tension de vapeur sont dits «tempérés ", c'est-à-dire que si la pression est contrôlée dans une enceinte par l'ouverture d'un évent, la température est également contrôlée, elle n'augmente pas et les réactions chimiques n'accélèrent pas s'il s'agit de réactions présentant des cinétiques d'Arrhénius d'ordre $n$ (pas d'autocatalyse).

Ces caractéristiques permettent généralement un contrôle aisé des emballements de réactions. La surface d'évent nécessaire est généralement moindre que pour les deux autres catégories de systèmes réactionnels. De plus, pour les systèmes à forte tension de vapeur, la pression dans les enceintes ne dépend pas du taux de remplissage.

Pour ces systèmes, la surface d'évent nécessaire est calculée à partir de la vitesse d'augmentation de température adiabatique mesurée à la température $T_{S}$ d'ébullition du mélange réactionnel, sous la pression $P_{S}$ d'ouverture de l'évent. Les données suivantes sont donc nécessaires :

- la vitesse d'augmentation de température adiabatique en fonction de la température,

- la courbe $P=f(T)$ correspondant à l'équilibre liquide vapeur.

L'évent sera traversé par un écoulement diphasique vapeur/liquide propulsé par la vaporisation.

Un exemple de système à tension de vapeur est l'emballement de la réaction Phénol + Formaldéhyde pour lequel la pression est la tension de vapeur de l'eau [2].

\subsubsection{Réaction produisant des gaz incondensables}

Il s'agit de réactions en phase condensée qui produisent des gaz incondensables comme $\mathrm{CO}, \mathrm{CO}_{2}, \mathrm{~N}_{2}, \mathrm{NO}, \mathrm{N}_{2} \mathrm{O}, \mathrm{O}_{2} \ldots \mathrm{La}$ pression dans l'enceinte est uniquement liée à la pression de ces gaz, il n'y a pas d'équilibre liquide/vapeur.

De ce fait, la température du système n'est pas contrôlable. Même si l'on parvient à limiter la pression dans l'enceinte par un évent, il n'y a pas de puits thermique de vaporisation, l'augmentation de la température n'est pas modifiée et les réactions chimiques accélèrent avec l'augmentation de la température.

Pour ces réactions, la pression dans l'enceinte dépend du taux de remplissage. Plus celui-ci est élevé, plus l'augmentation de la pression est importante et la vitesse d'augmentation de la pression élevée. Dans les tests de laboratoire utilisant des cellules de mesure fermées, le taux de remplissage doit être limité pour éviter l'éclatement de la cellule de mesure. De ce fait, les conditions expérimentales sont éloignées de l'adiabatique et il est préférable de recourir à des tests en cellule ouverte pour éviter cet inconvénient.

Le dimensionnement d'évents pour le contrôle de réactions produisant des gaz incondensables est basé sur la vitesse maximale de production de gaz mesurée dans des conditions adiabatiques. Il est en effet conservatif de considérer que cette vitesse sera atteinte même si l'évent est activé et de dimensionner ce dernier sur cette hypothèse.

L'évent sera traversé par un écoulement diphasique gaz/liquide propulsé par la détente des gaz incondensables.

Un exemple de réaction produisant des gaz incondensables et ne présentant pas de tension de vapeur est la décomposition d'un peroxyde lourd éventuellement dissout dans un solvant lourd.

\subsubsection{Systèmes réactionnels hybrides}

Un système hybride est un système réactionnel pour lequel la pression dans une enceinte est liée simultanément à une loi de tension de vapeur et à la production de gaz incondensables. Pour ces systèmes, la pression dans les enceintes dépend du taux de remplissage.
Par suite de l'équilibre liquide/vapeur, la surface d'évent est évaluée en tenant compte de la vitesse d'augmentation de la température et de la vitesse de production de gaz incondensables adiabatiques à la température d'ébullition du mélange réactionnel $T_{S}$ sous la pression d'ouverture de l'évent $P_{S}$.

L'évent sera traversé par un écoulement diphasique gaz/liquide propulsé simultanément par la détente de gaz incondensables et par la vaporisation.

Un exemple de système hybride est la décomposition de l'eau oxygénée $25 \%$ qui pressurise les enceintes par production d'oxygène et par tension de vapeur simultanément.

Les données de base mentionnées ci-dessus peuvent être obtenues en réalisant des tests de calorimétrie pseudo-adiabatique représentatifs des conditions de l'emballement thermique. Ces tests doivent être réalisés dans des conditions proches de l'adiabatique, dans des appareils semblables au Vent Sizing Package (V.S.P).

Une description des techniques calorimétriques pseudoadiabatiques utilisables pour le calcul d'évent est donnée dans la référence [3].

Une méthode simple de traitement des données de calorimétrie pseudo-adiabatique destinées au calcul d'évent est décrite dans la référence [4]. La technique de traitement décrite est pratiquement incontournable et généralement utilisée.

\subsection{Détermination de la surface d'évent idéale}

Il s'agit de la surface d'évent permettant de protéger l'enceinte sur laquelle elle est disposée mais sans tenir compte des pertes de charges des conduites situées en aval.

\subsubsection{Systèmes à forte tension de vapeur}

Pour les systèmes à forte tension de vapeur, la formule de Leung [5] est généralement utilisée :

$$
\begin{aligned}
& A=\frac{m o \cdot \bar{q}}{G\left[\left(\frac{V}{m o} \cdot \frac{h_{v l}}{V_{v l}}\right)^{1 / 2}+\left(C_{p} \cdot \Delta T\right)^{1 / 2}\right]^{2}} \\
& \bar{q}=\frac{1}{2} C_{P}\left[\left(\frac{d T}{d t}\right)_{s}+\left(\frac{d T}{d t}\right)_{m}\right]
\end{aligned}
$$

Le dimensionnement de l'évent requiert donc l'évaluation du flux massique $G$ de l'écoulement diphasique.

Une hypothèse conservative relativement à la protection de l'enceinte consiste à supposer que l'enceinte et l'écoulement présentent un comportement homogène. On a généralement recours à une méthode analytique et graphique publiée par J. Leung connue sous le nom de méthode Oméga du nom du paramètre de corrélation utilisé [5].

Cette méthode est basée sur le modèle HEM. L'évent est considéré comme une tuyère idéale. Le fluide diphasique rejeté est un fluide compressible. De ce fait, l'écoulement considéré peut présenter un régime critique pour lequel le flux massique ne dépend plus que des conditions de stagnation amont. On s'arrange pour placer l'évent dans des conditions d'écoulement critique, ce qui est généralement possible pour les écoulements diphasiques propulsés par la vaporisation.

\subsubsection{Réactions produisant des gaz incondensables}

La surface d'évent nécessaire est déterminée par la formule de Boyle basée sur un bilan volumique :

$$
\mathrm{A}=\frac{\mathrm{Q}_{\mathrm{gaz}, \max }^{\circ}}{\mathrm{G} \cdot \mathrm{V}}
$$


où $Q^{\circ}{ }_{\text {aaz, max }}$ est la vitesse maximale de production de gaz volumique, évaluée dans les conditions de l'enceinte au moment du rejet et $V$ le volume spécifique du fluide diphasique dans les mêmes conditions.

Le flux massique $G$ est évalué au moyen d'un modèle dérivé de la méthode $\omega$ pour les écoulements propulsés par la détente de gaz incondensables. Dans ce modèle le paramètre $\omega$ est identifié avec la fraction volumique moyenne de phase gazeuse dans l'enceinte. La version graphique du modèle est généralement utilisée. Elle permet également de déterminer les conditions de criticité de l'écoulement [5].

\subsubsection{Systèmes hybrides}

Pour les systèmes hybrides, la surface d'évent peut être évaluée par la méthode appropriée pour le comportement dont le système se rapproche le plus, système à forte tension de vapeur ou réaction produisant des gaz incondensables.

Plus récemment une méthode de calcul spécifique pour les systèmes hybrides tenant compte des débits de production de gaz et de vaporisation observés a été proposée par J. Leung [5]. Le calcul de la surface d'évent idéale pour les systèmes hybrides requiert le calcul du flux massique diphasique de l'écoulement hybride à travers le disque de rupture ou la soupape. Il est cette fois impossible de recourir à une méthode graphique pour déterminer $G_{C}$ et le rapport de pression critique car ces grandeurs dépendent de trop nombreux paramètres.

Un logiciel VSSPH permet de faire les calculs de dimensionnement d'évents sans avoir à manipuler ces calculs assez complexes.

\subsubsection{Influence des conduites en aval de l'orifice de l'évent}

Les pertes de charge dans les conduites en aval de l'organe d'obturation réduisent l'efficacité de l'évent installé. Pour compenser cet effet, il est nécessaire de corriger la surface d'évent idéale par un facteur $G_{C} / G_{O C}$ tenant compte de ces pertes de charge. Ce facteur peut être déterminé graphiquement en fonction de la valeur du paramètre $\omega$ et du facteur $4 f L / D$ de la conduite, pour les écoulements propulsés par la vaporisation et pour les écoulements diphasiques propulsés par la détente de gaz incondensable.

Pour les écoulements hybrides, on aura recours à des calculs numériques.

Dans les calculs relatifs à l'influence de la ligne d'évent sur la capacité de celui-ci, on tient compte du changement d'élévation entre l'entrée et la sortie de la ligne d'évent et de la présence de coudes ou de rétrécissements.

L'influence du changement d'élévation est prise en compte par un facteur d'inclinaison de l'écoulement $\mathrm{Fi}$.

L'influence des coudes et autres items sur la ligne est prise en compte par des incréments dans le facteur $4 f L D$ de la conduite.

\subsubsection{Avantages et inconvénients de la méthode de dimensionnement d'évent utilisant la méthode Oméga}

La méthode de calcul exposée ci-dessus a connu un certain succès car elle permet de réaliser les calculs de manière relativement simple à partir de données accessibles par l'expérimentation. Le calcul effectué présente un caractère conservatif car il est basé sur l'hypothèse d'un comportement homogène à l'équilibre thermodynamique dans l'enceinte à protéger, l'organe d'obturation et la ligne d'évent en aval.

Le calcul effectué ne vise pas à représenter la réalité physique de l'écoulement, les phénomènes d'écart à l'équilibre thermodynamique, les phénomènes non homogènes dans l'écoulement tels que :
- des désengagements gaz/liquide dans l'enceinte

- une brusque non homogénéité à l'entrée de l'évent par rapport à la situation dans l'enceinte,

- les phénomènes non homogènes dans les conduites en aval de l'organe d'obturation tels que glissement des phases liquide et gazeuse, écoulement stratifié, slug-flows, phénomènes oscillatoires.

D'une manière générale, les phénomènes hydrodynamiques de non-équilibre, le glissement des phases ou la stratification tendent à augmenter le flux massique de l'écoulement diphasique et donc l'efficacité de l'évent pour la protection de l'enceinte amont.

Il est concevable que les méthodes de calcul des écoulements diphasiques utilisées paraissent irréalistes à des spécialistes des écoulements diphasiques. L'utilisateur qui se livre à un calcul d'évent retient au contraire le côté pratique de ces méthodes, le fait que la méthode ne réclame que des données accessibles dans un laboratoire de sécurité des procédés correctement équipé et le fait que l'on parvient sans trop de problèmes au bout des calculs.

\subsubsection{Logiciels de dimensionnement d'évent et de simulation}

Le plus ancien de ces logiciels est le programme SAFIRE mis au point par H.K. Fauske pour le DIERS.

Le logiciel DEERS, mis au point par la société JAYCOR pour la marine américaine dans le but de calculer des tuyères de fusées utilisant l'hydrogène et l'oxygène comme carburant, n'a eu aucun succès pour le calcul d'évents.

Le logiciel Relief mis au point par le Centre Commun de Recherches d'Ispra de la Communauté Européenne n'a pas eu de succès commercial malgré certaines qualités ergonomiques.

Plus récemment, le logiciel BST proposé par le GIPH de Saint Petersbourg a été proposé.

Aux Etats-Unis, le logiciel SUPERCHEM a été proposé par Georges Melhem pour remplacer SAFIRE dans les méthodes du DIERS.

L'utilisation de ces logiciels est limitée par l'impossibilité de se procurer les données nécessaires à leur utilisation. C'est le cas pour SAFIRE et RELIEF.

De ce point de vue les logiciels proposant, en amont, des programmes d'acquisition des données nécessaires présentent un avantage décisif.

BST du GIPH admet comme données d'entrée, des cinétiques fournies par des logiciels de traitement de données obtenues par les méthodes calorimétriques courantes: ATD, calorimètre Setaram C80, essai en vase Dewar, essai en ARC, essai en VSP. Le logiciel BST est plus faible sur le calcul des équilibres thermodynamiques puisqu'il utilise un choix de solvants représentatifs qui peut ne pas convenir. L'utilisation de BST requiert néanmoins un effort très important sur le plan expérimental et pour l'obtention de données cinétiques représentatives.

SUPERCHEM est un logiciel thermodynamique très simplificateur pour ce qui concerne l'information cinétique. On peut s'attendre à ce que SUPERCHEM soit très bon sur le calcul des équilibres de phases. Pour le reste, nous manquons de recul.

\section{III —CAPTAGE ET TRAITEMENT DES REJETS D'ÉVENTS}

Les méthodes du DIERS disponibles ont pour avantage de permettre la protection des enceintes contre les conséquences d'un emballement thermique. Leur point faible est que le captage et le traitement du rejet ont été peu étudiés. Cela s'explique par le fait qu'aux Etats-Unis, il était permis de 
rejeter le contenu d'un réacteur dans l'environnement. La plupart des systèmes d'évents se limitent à une soupape ou un disque de rupture sans dispositif de captage ou de traitement du rejet réaliste à moins d'une toxicité sévère particulièrement évidente du rejet.

De ce fait, un très grand nombre d'évents de ce type sont installés sous la pression des assureurs puisque leur coût est limité.

La situation est différente en Europe où la densité de la population et des considérations relatives à l'environnemen conduisent à se préoccuper du traitement des rejets. Les accidents du Bhopal, Seveso, Griesheim et bien d'autres montrent la nécessité de tels dispositifs lorsque l'on installe un évent pour le contrôle d'un emballement thermique.

Le coût des dispositifs de traitement des rejets, leur encombrement et les problèmes de fiabilité qu'ils posent sont tels que l'on limite le recours à une protection par évent. On lui préfère la prévention des emballements de réactions par la mise au point de procédés fiables et l'utilisation de protections instrumentales redondantes.

\subsection{Dispositifs de captage des rejets envisageables}

Considérons maintenant le problème du choix et du dimensionnement des dispositifs de captage et de traitement des rejets d'évents.

Peu d'utilisateurs des méthodes du DIERS ont pris conscience que les méthodes de calcul adoptées pour le dimensionnement des évents et des conduites avals sont conservatives pour la protection de l'enceinte à protéger mais ne sont pas conservatives pour le dimensionnement des installations de captage du rejet en aval. Le modèle HEM et l'hypothèse homogène ont conduit à l'évaluation du plus faible flux massique envisageable.

Pour le dimensionnement des installations aval, il faut déterminer le flux massique le plus élevé envisageable et la composition du rejet diphasique en phase gaz et phase liquide.

Cela requiert des hypothèses différentes du comportement homogène dans l'enceinte et du modèle HEM pour les écoulements. Des modèles plus réalistes de désengagement gaz/liquide dans l'enceinte et d'écoulement diphasique dans les conduites d'évent doivent être utilisés. La transition des propriétés de l'écoulement à l'entrée de l'évent doit être prise en compte.

\subsubsection{Cyclones}

Il est difficile de dissuader les concepteurs de telles installations d'avoir recours à des séparations par cyclone. Les évaluations faites conduisent toujours à des cyclones très gros dont le diamètre peut être 5 fois celui du réacteur protégé. Des méthodes de dimensionnement de cyclones pour la séparation de rejets diphasiques gaz/liquide ont été publiées. On peut se reporter aux publications de Muschelknautz [6] et Grossel [7].

La séparation du liquide dans le cyclone doit être suivie d'un traitement de gaz en principe dans une tour d'absorption. Il est aussi nécessaire de contrôler les réactions chimiques dans le liquide séparé en le dirigeant vers une cuve de Quench.

\subsubsection{Cuve de Quench}

Le dimensionnement de cuves de Quench est probablement une technique plus simple que celle d'un ensemble Cyclone + Scrubber. Outre le volume de la cuve et la nature du liquide de Quench, il y a lieu de choisir le système d'injection du rejet diphasique dans le liquide de Quench :

- simple tube plongeant.
- queue de carpe,

- râteau,

- éjecteurs.

On notera le système d'éjecteurs proposé par Klaus Hermann permettant de créer une aspiration du liquide de Quench dans l'éjecteur et un bon mélangeage du rejet diphasique avec le liquide de Quench [8]. Ce dispositif a reçu une subvention de la CEE.

\subsubsection{Séparation liquide/vapeur et réinjection du liquide dans le réacteur}

Dans ce dispositif [9], le rejet diphasique résultant de la dépressurisation d'un réacteur est envoyé dans un séparateur à choc. Le liquide séparé retombe dans une conduite reliée au réacteur par une vanne. Lorsque le niveau dans la conduite est suffisant, celle-ci est mise en relation avec le réacteur dans lequel elle se vide.

Ce dispositif astucieux est intéressant pour les systèmes à tension de vapeur car le liquide renvoyé dans le réacteur se trouve refroidi par la vaporisation et fournit un puits thermique favorisant le contrôle de la réaction.

L'avantage de ce dispositif est qu'il n'y a pas émission de mélange réactionnel vers l'extérieur. Celui-ci est renvoyé dans le réacteur par un système peu coûteux.

L'auteur [9] démontre l'efficacité du système en réalisant, dans une installation pilote, des dépressurisations sur des charges d'eau sous-refroidie. Ce dispositif aurait été installé sur un gros réacteur pour la fabrication de latex.

\section{CONCLUSION}

Dans les pays à forte densité de population, où l'industrie chimique se trouve implantée dans des zones urbaines, aucun rejet de mélange réactionnel n'est admis en cas d'incident lié à un emballement thermique. Cet objectif peut être atteint essentiellement par des mesures préventives qui permettent d'éliminer les conditions de procédé dangereuses. Des exemples représentatifs de ces mesures préventives sont :

- l'élimination des procédés en batch total et leur remplacement par des procédés semi-continus ou continus,

- l'élimination des procédés de concentration ou d'évaporation en batch et leur remplacement par des processus continus,

- l'élimination des procédés fonctionnant sous pression élevée,

- l'étude approfondie des procédés et de leurs dérives possibles afin de prévenir les accidents. Cette étude comprend les étapes suivantes :

* recherches bibliographiques sur le procédé et les réactions en cause. Recherche des accidents connus sur le procédé,

* étude expérimentale du procédé, des réactions mises en œuvre et des dérives possibles du procédé,

* étude des dérives possibles par la méthode HAZOP, s'appuyant sur les résultats de l'étude bibliographique et de l'étude expérimentale du procédé.

- L'instrumentation du procédé et l'élimination des dérives dangereuses par des sécurités instrumentales redondantes.

Le risque résiduel peut alors être traité par des évents de taille plus faible et collectés.

Pour les évents existants, il y a lieu de réexaminer leur nécessité, vérifier leur dimensionnement, diriger le rejet attendu vers une installation de captage appropriée. Il est souvent possible d'éliminer les dérives ayant conduit à l'installation de l'évent par un aménagement du procédé ou l'installation de sécurités instrumentales.

Le retour d'expérience sur le fonctionnement des évents installés dans la période récente est faible car les procédés 
correctement étudiés, instrumentés et protégés n'ont pas d'accident.

La référence à des pratiques ayant cours aux Etats-Unis, où la collecte des évents n'est pas requise sur une base réglementaire, n'est pas adaptée au contexte européen. On peut opposer de ce point de vue la politique du «toutévent » suivie aux Etats-Unis, où l'installation d'un évent est demandée mais sans la collecte du rejet, à la politique du zéro rejet pratiquée en Europe et particulièrement en Allemagne après l'accident de Griesheim (1993). Le contexte européen favorise à l'évidence une politique de prévention des accidents basée sur l'étude des procédés et la protection instrumentale.

\section{RÉFÉRENCES}

[1] FISHER H.G. (1985). - "DIERS, an overview of the program«, Loss Prevention Symposium, AIChE Houston National Meeting.

[2] GUSTIN J.L., FILLION J., TREAND G., EL BIYAALI K. (1993). - The Phenol + Formaldehyde Runaway Reaction, Vent Sizing for Reactor Protection. J. Loss Prev. Process Ind, vol. $6, \mathrm{n}^{\circ} 2,103-113$.
[3] GUSTIN J.L. - Calorimetry for Emergency Relief Systems Design, Eurocourse, Reliability and Risk Analysis, vol.1, Safety of Chemical Batch Reactors and Storage Tanks, 311-354, Kluwer Academic Publishers.

[4] GUSTIN J.L. (1995). - « Assessment of Runaway Reaction Hazards using the experimental Methods of the Process Safety Laboratories ", International Symposium on Runaway Reactions and Pressure Relief Design, Boston, August 2-4, 150-185.

[5] LEUNG J.C. (1995). - « Simplified Vent Sizing Methods Incorporating two-phase Flow ", International Symposium on Runaway Reactions and Pressure Relief Design, Boston, August 2-4, 200-236.

[6] Muschelknautz S. and MAYINGER F. (1990). — « Separation of liquid from Vapour upon Pressure Relief ", J. Loss Prev. Process Ind., vol. 3, January, 125-129.

[7] GROSSEL S.S. (1990). - « An overview of equipment for containment and disposal of Emergency Relief Systems Effluents ", J. Loss Prev. Process Ind., vol. 3, 112-124.

[8] HERMANN K., ROGERS R. (1995). - International Symposium on Runaway Reaction and Pressure Relief Design, Boston, August 2-4, 743-764.

[9] MOLTER E. (1992). - « Reintroduction of Liquid phase into the reactor after Separation from a two-phase Flow during Pressure Relief ». 7th International Symposium on Loss Prevention and Safety Promotion in the Process Industries, Taormina, Italy, 4-8 May, Paper $n^{\circ} 125$. 